\title{
Comparison of a Developed Dual Axis Solar Tracking System based on Maximum Power Point Tracking (MPPT) and Astronomical Equations with Existing Systems
}

\author{
Anuja Borkar \\ Mumbai University \\ Fr.Agnel Institute of Technology, Bandra
}

\author{
Sunil Surve, $\mathrm{PhD}$ \\ Mumbai University \\ Fr.Agnel Institute of Technology, Bandra
}

\begin{abstract}
A novel, low cost solar tracker based on Maximum Power Point Tracking (MPPT) and astronomical equation is designed and implemented. Simulated results of the system predict an increase in efficiency compared to other tracking solar panels. Experimental testing also shows an excellent agreement with the available models. In addition, further improvements to the design are critically evaluated in terms of complexity and benefit.
\end{abstract}

\section{Keywords}

Solar tracking, efficiency, maximum power point tracking (MPPT), astronomical equations

\section{INTRODUCTION}

A considerable progress has been made over the last few decades for development of renewable energy systems such as wind, sea wave and solar energy systems.

Among all of these resources, solar or photovoltaic energy is considered as most reliable, as it can be procured on daily basis as well as environment friendly renewable source of energy $[1,2]$. But, solar energy systems generally suffer from their low efficiencies in delivering the output and also of its high costs for the implementation of panel [3]. In order to attain higher efficiency, maximum power should be extracted from these systems using either physical tracking or maximum power point tracking (MPPT).

Physical tracking deserves the aligning of the photo-voltaic (PV) panels to be orthogonal direction to the sun rays throughout the day in order to achieve maximum solar radiation [4-11], usual manual or automatic tracking is done in this case. In manual tracking, the PV panel orientation is changed manually at the starting of each season to a predefined angle. But, in case of automatic tracking, the PV panel is mounted on a single-axis or multi-axis tracking mechanism and is operated to follow the sun rays trajectory throughout the day [4-11]. The mechanisms of tracking can be classified into three types, basically, passive method, optical method, and the astronomical method. The general features that contribute to an efficient tracking mechanism are: capability to extend over a wide range of space, compactness against wind disturbances, and lastly the minimum power consumption.

Passive tracking depends on the heating properties of gas matters such as Freon et al. [5]. The tracker consists of two cylindrical tubes fitted on the edges of the panel; under partial pressure these tubes are filled with fluid. The radiation from the sun will increase the fluid volume to expand in the direction of the sun. So, the cylinder gas pressure will cause an unbalance in the tracker. As a result, the tracker will tilt to the sun side. This type of system is mainly cheap and needs little maintenance. However, because of its lower efficiency at low temperatures it is not well established and accepted by the consumers [4].

The second method of tracking deals with the optical or electro-optic trackers. This method uses different feedback sensors such as photo-sensors, current, voltage sensors, and auxiliary cells to figure out the panel reference position [6-8]. Mainly fuzzy logic or PID closed-loop position control systems are used to produce the actuators control commands. The disadvantage for such a system is that it is very susceptible to atmospheric conditions (clouds shading) and might not be able to continue tracking the sun in a cloudy day.

The third tracking method is the astronomical method, which incorporates the longitude and latitude data of a given location to relate to the current position of the sun [9-11]. Simpler programming, reduced implementation cost, and lower power consumption as the need for additional sensors is eliminated which are the main advantages of the optical method. This method also delivers high degree of accuracy and also is not sensitive to atmospheric conditions. In addition, for optimization of the efficiency of PV systems MPPT is used. MPPT is a real-time control scheme applied to the PV system charger in order to extract the maximum power possible from the PV panel.

The MPPT working principle depends on the maximum power point tracking. The power delivered from the source to the load is maximized when the input resistance seen by the source matches the source resistance. Therefore, in regard to transfer maximum power from the panel to the load the internal resistance of the panel has to equal the resistance seen by the PV panel. For a fixed load, the resistance seen by the panel can be adjusted by changing the charger (DC/DC converter) duty cycle [12-14].

In this paper, an attempt to combine advantages of MPPT and astronomical equation based sun tracking system is made and is compared to other existing tracking systems.

\section{PROPOSED SYSTEM}

The block diagram of a proposed system is presented below in fig. 1 . The system is powered by two sources, solar power or battery depending up on higher voltage. 


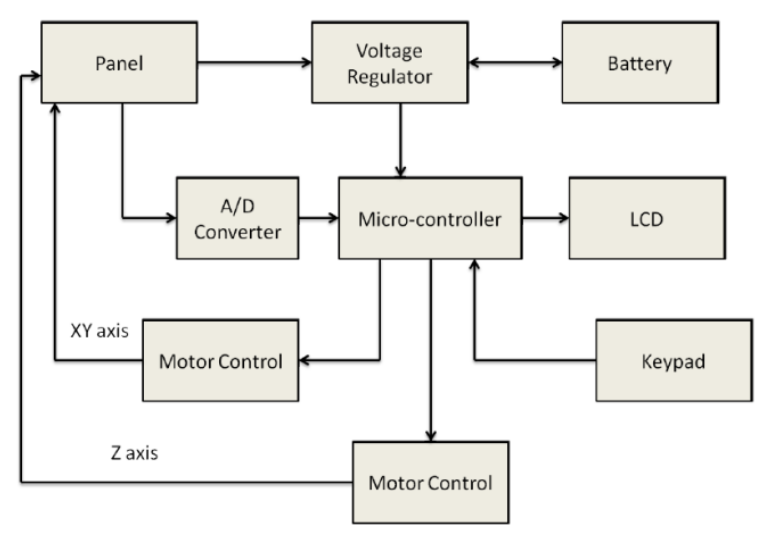

Figure 1. Block diagram of proposed system

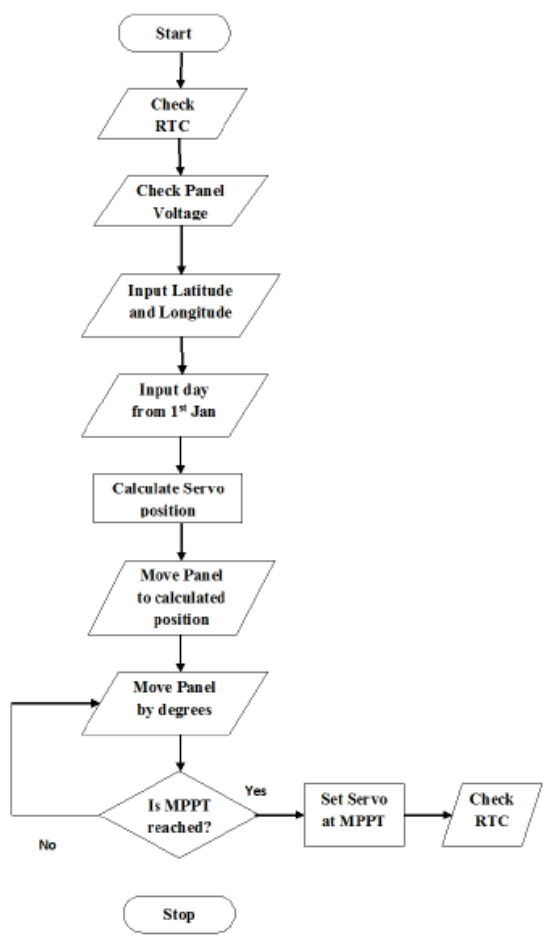

Figure 2. Flow chart of the proposed system

The panel voltage and current are measured through an analog to digital converter (ADC). Rotation of the panel is controlled by servomotor and LCD is used to display the current values such as longitude, latitude etc. Keypad of $4 \times 4$ is used to input the values and to interface the system manually.

The flow chart implemented to run the system is provided fig. 2. Initially the Real Time Clock (RTC) is set from an input keyboard which is alphanumeric keypad. Once the RTC is set, the system checks panel voltage via the ADC. Once the panel voltage is known, system asks the user for longitude and latitude entry. Depending up on astronomical equations, solar panels exact angles are computed named as polar and tilt angle. User then will be prompted to enter day with respect to 1 st January and the servo motors will be rotated to correct positions as per the astronomical equations. Depending upon MPPT point, the panel finally settles at a desired position. To determine this MPPT point panel actually moves 20 degrees in both positive and negative direction from its current position. In this way the system is executes its operation.

\section{RESULTS AND DISCUSSION}

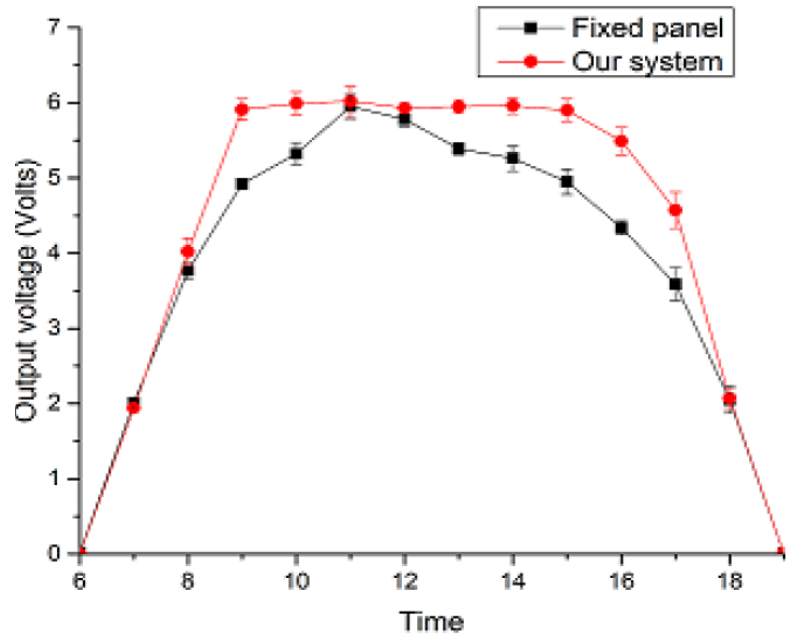

Figure 3. Plot of output voltage vs. time of our's compared with the fixed panel system

Fig. 3 shows that the average output voltage vs time of the day (in 24 hours clock format). It can be seen from the graph that our system is provides throughout better output voltage for same load current except two to three data points in initial phase and ending phase of measurement.

In sense of costing if it is compared to other systems, it shows (in Fig. 5) that the cost for our system per rupee is approximately $420 \mathrm{mWatt}$ of power. Hence to deploy entire system of 1000 Mega Watt power station estimated cost will be around 4.2 Cr. INR rupees. From companies existing market survey done in Mumbai, the cost estimate obtained for MPPT were $4.8 \mathrm{Cr}$. INR, which is way higher than our system. It is found by analysis that single axis tracking and astronomical equation based single axis tracking will cost less compared to our system.

\section{CURRENTLY IN USE VS OUR SYSTEM}

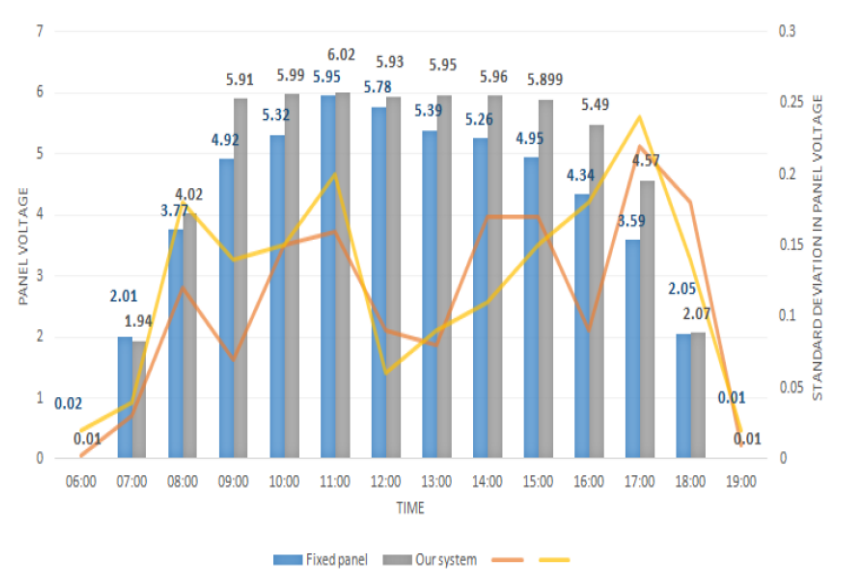

Figure 4. Plot showing the panel voltage (volts) vs. time(Hr) of currently used vs. our system comparison 


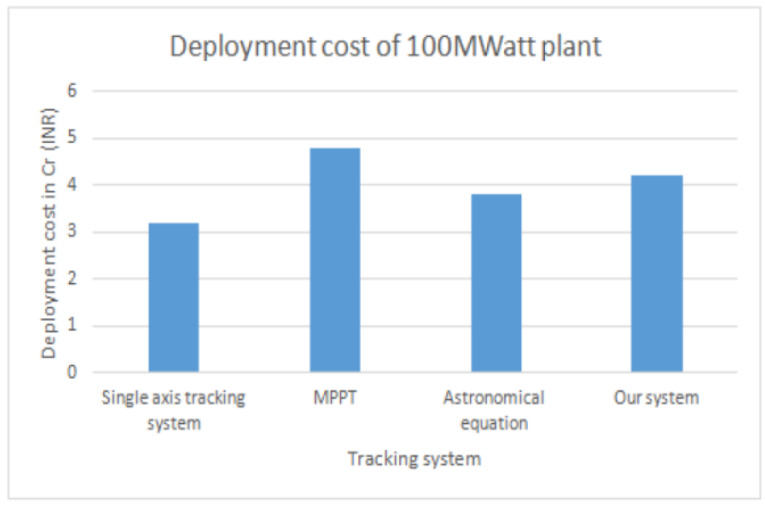

Figure 5. Bar graph plot showing the development cost (in crore) of the existing tracking system with our system.

SYSTEMS VS DIFFERENT POWERS

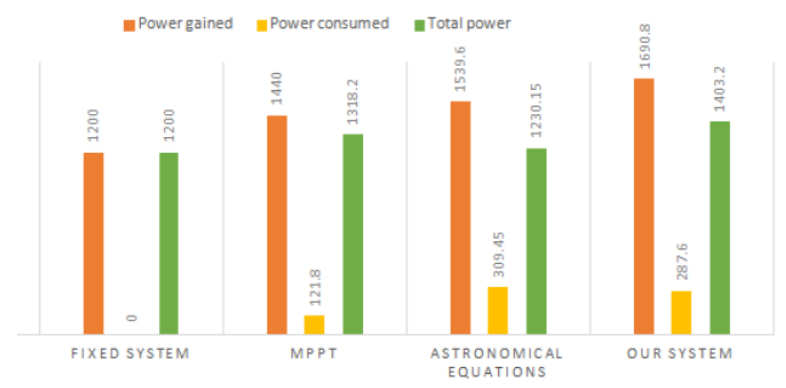

Figure 6. Bar graph plot showing power consumed, power gained and total power of different systems.

The comparison of our system with the other system in sense of power consumption vs. power gained is also studied. It is seen (in Fig. 6) that the proposed system consumes less power as compared to the other dual axis systems such as Azimuthaltitude single axis tracker (AASAT), astronomical equation based tracker. But the power consumed in rotating the panel is more than only MPPT and fixed panel system. When computed the difference between power gained by moving the panel and power lost in moving the panel of our system, proves that it is the best although individually in power gain as well as power lost system is estimated to be the second best.

Fig. 6 shows the bar graph plot of different system vs. powers consumed, gained and total power (i.e. the difference between the power gained by moving the panel and power lost in moving the panel). It is observed from the plot that our system can effectively earned $1403 \mathrm{~mW}$ power as compared to 1200 $\mathrm{mW}$ for fixed panel as well as with MPPT and astronomical equation based trackers running individually can go maximum upto $1318 \mathrm{~mW}$ and $1230 \mathrm{~mW}$ respectively.

When different systems are compared in the basis of normalized power it is seen (in Fig. 7) that the proposed system has a normal power value of $\sim 140$.

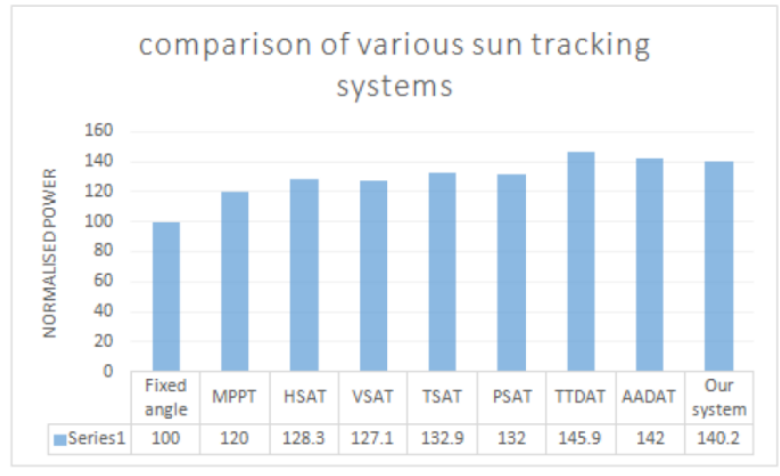

Figure 7. Bar graph plot comparing various tracking system with respect to normalized power.

\section{CONCLUSION}

Our system is an attempt to combine advantages of MPPT and astronomical equation based sun tracking system. As compared to fixed panel system our system of sun-tracking always give more power except initial and final one or two points. System is right now in initial phase and did not made it as good as Azimuth-altitude dual axis tracker (AADAT) and Tip-Tilt Dual Axis Tracker (TTDAT) just by 2\% but already proved better as compared to fixed panel, only MPPT, horizontal single axis trackers (HSAT), vertical single axis trackers (VSAT), tilted single axis trackers (TSAT) and polar aligned single axis trackers(PSAT) system.

By rotating the panel in two axis $20-40 \%$ power were improved. Power lost in rotating panel is much higher than what we get by rotating panel if convergence to MPPT does not happen at enough proportion. Our system does not consume power as much as astronomical equation based continuous tracking. Total power received by combination of MPPT and astronomical equation based system is 10-20\% higher than individual systems acting standalone.

The proposed system is low cost as compared to be more accurate Azimuth-altitude dual axis tracker (AADAT) and tilted angle dual axis tracker (TADAT) system. The deployment of $1000 \mathrm{MW}$ would be approximately around 4 Cr. INR. Thus the idea for developing this type of solar tracker with higher efficiency is always welcome and would be a great boon in the near future.

\section{ACKNOLEDGEMENT}

I would like to thank the Almighty.I am sincerely thankful to my Respected Parents, Dear Husband and family for their tremendous support throughout the process. Special thanks to my guide for the immence guidance and motivation.I am Ambadnya.

\section{REFERENCES}

[1] G. Spagnuolo, G. Petrone, S. V. Araujo, C. Cecati, E. Friis-Madsen, E.Gubia, D. Hissel, M. Jasinski, W. Knapp, M. Liserre, P. Rodriguez, R.Teodorescu, and P. Zacharias, "Renewable energy operation and conversion schemes," IEEE Ind. Electron. Mag., vol. 4, no. 1, pp. 38-51, Mar. 2010.

[2] P. Fairley, "Fukushima's positive impact [spectral lines]," IEEE Spectrum,vol. 48, no. 5, p. 8, May 2011. 
[3] B. Bose, "Global warming: Energy, environmental pollution, and the impact of power electronics," IEEE Ind. Electron., vol. 4, no. 1, pp.6-17, Mar. 2010.

[4] H. Mousazadeh, A. Keyhani, A. Javadi, and H. Mobli, "A review of principle and sun-tracking methods for maximizing solar systems output," Renewable and Sustainable Energy Reviews, vol. 13, pp.1800-1818, 2009.

[5] M. Clifford and D. Eastwood, "Design of a novel passive solar tracker," Solar Energy, vol. 77, pp. 269280, 2004.

[6] R.-J.Wai, W.-H.Wang, and C.-Y. Lin, "Highperformance stand-alone photovoltaic generation system," IEEE Trans. Ind. Electron., vol. 55,no. 1, pp. 240-250, Jan. 2008.

[7] C. Chin, A. Babu, and W. McBride, "Design, modeling and testing of a standalone single axis solar tracker using MATLAB/Simulnik,"

[8] Renewable Energy, vol. 36, pp. 3075-3090, 2011.

[9] [8] H. Lu and T. Shih, "Fuzzy system control design with application to solar panel active dual-axis Sun tracker system," in Proc. SMC'10, Istanbul, 2010, pp. 1878 1883.

[10] S. Abdallah and O. Badran, "Sun tracking system for productivity enhancement of solar still," Desalination, vol. 220, pp. 669-676, 2008.

[11] S. Seme, G. “Stumberger, and J. Vor`si`c, "Maximum efficiency trajectories of a two-axis sun tracking system determined considering tracking system consumption," IEEE Trans. Power Electron., vol. 26, no. 4, pp. 12801290, Apr. 2011.

[12] A. Al Nabulsi, A. El Nosh, A. Ahli, M. Sulaiman, and R. Dhaouadi, "Efficiency optimization of a $150 \mathrm{~W}$ PV system using dual axis tracking and MPPT," in Proc. IEEE ENERGYCON'10, Bahrain, pp. 400-405.

[13] E. Aranda, J. Galá N, M. Cardona, and J. Rquez, "Measuring the i-v curve of PV generators," IEEE Ind. Electron. Mag., vol. 3, no. 3, pp. 4-14, Sep. 2009.

[14] R. F. Coelho, F. M. Concer, and D. C. Martins, "Analytical and experimental analysis of DC-DC converters in photovoltaic maximum power point tracking applications," in Proc. IECON'10, Phoenix, AZ, 2010, pp. 2778-2783. 